\title{
Reduction of Halo Pin Site Morbidity with a New Pin Care Regimen
}

\author{
Hussain Anthony Kazi, Marcus de Matas, Robin Pillay \\ Spinal Unit, Department of Orthopaedics, Royal Liverpool University Hospital, Liverpool, UK
}

Study Design: A retrospective analysis of halo device associated morbidity over a 4-year period.

Purpose: To assess the impact of a new pin care regimen on halo pin site related morbidity.

Overview of Literature: Halo orthosis treatment still has a role in cervical spine pathology, despite increasing possibilities of open surgical treatment. Published figures for pin site infection range from $12 \%$ to $22 \%$ with pin loosening from $7 \%$ to $50 \%$.

Methods: We assessed the outcome of a new pin care regimen on morbidity associated with halo spinal orthoses, using a retrospective cohort study from 2001 to 2004. In the last two years, our pin care regimen was changed. This involved pin site care using chlorhexidene \& regular torque checking as part of a standard protocol. Previously, povidone iodine was used as skin preparation in theatre, followed by regular sterile saline cleansing when pin sites became encrusted with blood.

Results: There were 37 patients in the series, the median age was 49 (range, 22-83) and 20 patients were male. The overall infection rate prior to the new pin care protocol was $30 \%(n=6)$ and after the introduction, it dropped to $5.9 \%(n=1)$. This difference was statistically significant $(p<0.05)$. Pin loosening occurred in one patient in the group prior to the formal pin care protocol $(3 \%)$ and none thereafter.

Conclusions: Reduced morbidity from halo use can be achieved with a modified pin cleansing and tightening regimen.

Keywords: Cervical spine; Trauma; Tumour; Spinal injury; Orthosis

\section{Introduction}

The use of halo devices has become widespread, allowing a less invasive way to treat cervical spine instability due to trauma, tumour or ligamentous injury [1]. Devices may be used as primary treatment or as an adjunct to surgical stabilisation. The device allows early discharge, rehabilitation and mobilization. Perry and Nickel [2] first described the device in 1959 for control of a long paralytic curve in poliomyelitis. With time, its indications have expanded [3]. A high incidence of pin loosening, infection and discomfort, as well as pressure ulceration from the jacket were noted in a study performed in 1986 [3]. Other complications include lower respiratory tract infection/respiratory failure [4-7], neuropraxia [8], neuroma [9], brain abscess [10,11], epidural abscess [12], pneumocephalus, and hemiparesis [13] and epileptic seizure [14]. There have been isolated reports of myiasis (maggot infestation) [15], as well as depressed skull fracture and cerebral contusion after a fall onto the posterior pin [16]. Our inclination was that the incidence of complications is still true today and we tested this by auditing the results in our regional spinal unit.

Received Apr 12, 2012; Revised Jul 16, 2012; Accepted Aug 4, 2012

Corresponding author: Hussain Anthony Kazi

Spinal Unit, Department of Orthopaedics, Royal Liverpool University Hospital,

Prescot Street, Liverpool, L7 8XP, UK

Tel: +44(0)7733-335543, F: +44(0)151-706-5839, E-mail: huzzkazi@hotmail.com 


\section{Materials and Methods}

A retrospective cohort study of a consecutive series of patients was performed. All patients in whom a halo device was applied in our unit between January 2001 and December 2004 were included. Any patient transferred to us with an orthosis already applied was excluded.

During the final two years, our pin care regimen was altered to one adapted from that used by our limb reconstruction team for the care of fine wire external fixators. Prior to this, our in house protocol involved the use of povidone iodine at the time of halo application, followed by cleaning of pin sites with sterile saline if encrusted with blood. We aimed to assess if the adaptation of a published pin care regimen would reduce our pin site complication rate [17].

Patient details were sourced from the spinal register held in the department and then corroborated against the theatre database; any casenotes with a halo procedure listed were sourced and reviewed. Institutional review board approval was granted.

A complete dataset was achieved in 37 out of 38 patients (one case sheet had been destroyed). Age range was 22 to 83 years (median, 49 years; mean, 50.27 years), consisting of 20 males and 17 females.

Twenty-eight halos were applied under local anaesthetic (LA); one was applied with LA and sedation. The remaining 8 were applied under general anaesthetic (GA), 2 due to the need for another trauma procedure (1 tibial nailing, 1 femoral nailing) and 6 due to a Glasgow Coma Score of 8 or less. Lead surgeon was either consultant $(n=6)$, specialist registrar $(n=29)$ or senior house officer $(\mathrm{n}=2)$.

Halos were applied for fractures $(n=21)$, tumours $(n=6)$ and subluxations $(\mathrm{n}=10)$. Mechanism of injury (fractures and subluxations) included a road traffic accident $(n=9)$, seizure $(n=2)$, fall $(n=5)$, assault $(n=2)$, and skiing $(n=1)$. Alcohol consumption was involved in $47 \%$ as a factor. A demographic comparison is enclosed, as well as comorbidities, which were similarly distributed between the two groups (Table 1).

Fractures where at the level of C2 $(n=10)$, C6 $(n=5), C 4$ $(\mathrm{n}=4)$, C5 $(\mathrm{n}=4)$ C1 $(\mathrm{n}=3), \mathrm{C} 7(\mathrm{n}=2)$, and C3 $(\mathrm{n}=1)$. Subluxations were mostly at $\mathrm{C} 5 / 6(\mathrm{n}=7)$, followed by $\mathrm{C} 6 / 7$ $(\mathrm{n}=2)$, and $\mathrm{C} 4 / 5(\mathrm{n}=1)$. Tumours included metastases from breast $(n=1)$, bronchus $(n=1)$, colon $(n=1)$, gastric $(n=1)$, and unknown primary $(n=2)$. Duration of applica-
Table 1. Patient characteristics

\begin{tabular}{|c|c|c|}
\hline Variable & 2001-2002 & 2003-2004 \\
\hline \multicolumn{3}{|l|}{ Sex } \\
\hline Male & $11(55)$ & $10(59)$ \\
\hline Female & $9(45)$ & $7(41)$ \\
\hline \multicolumn{3}{|l|}{ Comorbidities } \\
\hline Diabetes & $4(20)$ & $3(18)$ \\
\hline Steroid use (COPD) & $4(20)$ & $4(24)$ \\
\hline Alcoholism & $10(50)$ & $7(42)$ \\
\hline Polytrauma & $7(35)$ & $7(42)$ \\
\hline Tumour (primary) & $3(15)$ & $3(18)$ \\
\hline \multicolumn{3}{|l|}{ Pathology } \\
\hline Fracture & $7(35)$ & $11(65)$ \\
\hline Subluxation & $10(50)$ & $3(18)$ \\
\hline Tumour (metastasis) & $3(15)$ & $3(18)$ \\
\hline \multicolumn{3}{|l|}{ Treatment } \\
\hline Halo alone & $13(65)$ & $13(76)$ \\
\hline Halo then surgery & $7(35)$ & $4(24)$ \\
\hline \multicolumn{3}{|l|}{ Duration of application } \\
\hline 1-7 day & $2(10)$ & $1(6)$ \\
\hline 7-28 day & $3(15)$ & $2(12)$ \\
\hline $1-2$ mo & $3(15)$ & $2(12)$ \\
\hline $2-3$ mo & $12(60)$ & $9(53)$ \\
\hline$>3 \mathrm{mo}$ & $0(0)$ & $3(17)$ \\
\hline \multicolumn{3}{|l|}{ Surgery } \\
\hline Fusion & $4(20)$ & $1(6)$ \\
\hline Vertebrectomy and cage & $2(10)$ & $0(0)$ \\
\hline $360^{\circ}$ stabilisation & $1(5)$ & $0(0)$ \\
\hline Posterior stabilisation & $0(0)$ & $3(17)$ \\
\hline Total & $7(35)$ & $4(24)$ \\
\hline
\end{tabular}

Values are presented as number (\%).

COPD, chronic obstructive pulmonary disease.

tion ranged from 5 to 126 days. The median duration was 84 days. There was no difference of duration dependent on the injury or level. Those with a short duration of application had early open surgery ( 3 patients had an application of 21 days or less).

One investigator reviewed all casenotes. Specific attention was paid to the operation notes, clinical entries and clinic letters. The microbiology computer system was reviewed for any positive results during the time period of halo application. Patient details were checked with consultant's personal databases and the departmental spi- 
nal register. Pin site infections were defined as a pin site that was painful or inflamed and discharging, either with positive cultures or a response to antibiotics [17]. Pin loosening was defined as either a pin that could be freely twisted by the examiner without resistance, when the pin tip was visible at the skin edge or one that measured less then $0.90 \mathrm{Nm}(8 \mathrm{inlb})$ of torque $[3,18]$. Data was entered into an Excel database (Microsoft, Redmond, WA, USA) and then reviewed. Statistical analysis was performed using the unequal variance $t$-test for independent samples [19].

Devices were applied in theatre using standard aseptic precautions. The application took place under either GA or LA, dependent on patient circumstances. All orthoses applied were Bremer HALO Crown with Classic II vest (Depuy Spine, Warsaw, IN, USA), using the operative technique advised by the manufacturer. Inline cervical stabilisation was maintained at all times and image intensifier was utilized. The patient was placed in a supine position, and using suction cups, the halo crown was applied in an adequate position avoiding adjacent anatomical structures (about $1 \mathrm{~cm}$ above eyebrows and tilted slightly above the horizontal plane when viewed laterally).

Skin was prepared using either povidone iodine (Videne, Ecolab, Leeds, UK) prior to 2003 or chlorhexidene solution (Hydrex, Ecolab) from 2003 onwards. Shaving was not routinely performed. Local anaesthetic ( $1 \%$ lidocaine with adrenaline 1 in 200,000, non-proprietary) was applied to raise a small bleb directly through the chosen hole in the halo crown into the skin and periosteum. The halo pins were then applied with one in each quadrant of the cranium leaving empty holes on either side to allow for extra pin placement if required (in cases of infection or loosening). Four pins were used routinely. Anterior pins were engaged above the supraorbital ridges over the lateral one third of the eyebrows in order to avoid the supratrochlear and supraorbital nerves and allow patients a clear field of vision. The posterior pins were placed at 90 degrees to the anterior pins. Opposite pins were tightened simultaneously to a torque of 0.90 $\mathrm{Nm}$. Once the ring was applied, the patient was log rolled, and the jacket and posts were applied. This was adjusted to give a secure fit, whilst allowing chest wall movements. Fine-tuning was performed in relation to the patient's head position and lateral radiographic appearances.

Prior to January 2003, pin care involved the use of povidone iodine dressings at the time of the operation, followed by cleaning with sterile saline if pin the sites became encrusted or dirty, pin torque was not routinely checked after application. After this date a new pin care regimen was introduced and adapted from that utilised by limb reconstruction surgeons [17]. The pin care regimen involved aseptic technique when handling the pins. Pins were inserted carefully to minimize skin trauma and any bone swarf (bone debris from pin insertion) was removed with saline lavage. Pins were immediately dressed with gauze soaked in alcoholic solution of chlorhexidene (Hydrex, Ecolab) with pressure to reduce bleeding, which was changed at the completion of the procedure if bloodstained. Pin sites were then cleaned daily for three days, using 70\% alcohol to ensure blood removal. Dressings moistened with Hydrex were then reapplied. Pin cleaning and redressing was repeated every 7 to 10 days, using the same technique. In the interim and after discharge, patients were encouraged to wash using $4 \%$ chlorhexidene gluconate (Hibiscrub, Regent Medical, Dunstable, UK) solution on a daily basis. Pin torque was checked every second day for the first week, using a $0.90 \mathrm{Nm}$ torque wrench, then weekly for the first month. Thereafter, torque was checked monthly until halo removal. The utilized torque was that recommended by the manufacturer.

\section{Results}

Complications were divided into the major and minor groups and detail of these is enclosed (Table 2). One patient with a significant head injury suffered intractable nausea and vomiting after halo application, but this was considered a sequela of the head injury itself, rather than halo application, and settled spontaneously after 5 days.

\section{Iodine group (2001-2002)}

Eight patients required pin repositioning. This was due to poor initial position in 2 patients, pain in 4 patients (13.5\%), pin loosening in one patient (3\%) and pin site infection in another. In these patients, adjacent holes were used for replacement pins to reduce the risk of skull penetration.

Pin site infections were defined as a pin site that was painful or inflamed and discharging, either with positive cultures or a response to antibiotics [17]. This occurred in 6 patients in this group, 5 were managed with antibiotics 
Table 2. Complications

\begin{tabular}{|c|c|c|}
\hline Variable & $2001-2002$ & 2003-2004 \\
\hline \multicolumn{3}{|l|}{ Major complications } \\
\hline Death & $0(0)$ & $2(12)$ \\
\hline \multicolumn{3}{|l|}{ Permanent deficit } \\
\hline Frankel A & $1(5)$ & $1(6)$ \\
\hline Frankel B & $0(0)$ & $0(0)$ \\
\hline Frankel C & $0(0)$ & $0(0)$ \\
\hline Frankel D & $1(5)$ & $3(18)$ \\
\hline \multicolumn{3}{|l|}{ Nerve palsy } \\
\hline Greater occipital & $0(0)$ & $0(0)$ \\
\hline Supraorbital & $0(0)$ & $0(0)$ \\
\hline Thromboembolism & $0(0)$ & $0(0)$ \\
\hline Pressure ulceration & $1(5)$ & $1(6)$ \\
\hline Respiratory impairment & $1(5)$ & $2(12)$ \\
\hline Seizure & $1(5)$ & $0(0)$ \\
\hline \multicolumn{3}{|l|}{ Minor complications } \\
\hline Pin penetration & $0(0)$ & $0(0)$ \\
\hline Pin loosening & $1(5)$ & $0(0)$ \\
\hline Painful pin & $4(20)$ & $3(18)$ \\
\hline Pin site infection & $6(30)$ & $1(5.7)$ \\
\hline Pin repositioning & $8(40)$ & $3(18)$ \\
\hline
\end{tabular}

Values are presented as number (\%).

and one with pin removal and repositioning. The pin site infection rate was $30 \%$.

All pin site bacteriological cultures revealed staphylococcus aureus, which was sensitive to penicillin (there were no methicillin resistant staphylococcus aureus isolates).

\section{Chlorhexidene group (2003-2004)}

Three patients required repositioning, all due to painful pin sites. There were no cases of pin loosening. Only 1 pin site became infected $(1 / 17,5.9 \%)$, which was managed with antibiotics alone. This difference was statistically significant $(p<0.05$, actual value $p<0.027)$.

\section{Discussion}

Our case series confirms the morbidity and mortality associated with halo use. The literature reveals widespread reports of numerous complications; the most common being pin loosening and pin site infection. Published rates of pin loosening range from $7 \%$ to $60 \%$, and for pin site infection from $12 \%$ to $22 \%$ [19]. Severe pin discomfort has been quoted in the region of $18 \%$; our rate of $18.9 \%$ is not significantly different $[3,4]$.

The most striking finding in this series was the reduction in pin site complications after using a regimen previously suggested for fine wire external fixators by Davies et al. [17]. This protocol was adapted for spinal use. Modifications include using eye protection, and an education programme for each patient carried out by a specialist spinal nurse. Instituted from January 2003, this was associated with a reduction in pin site infection, down from 6 patients $(6 / 20,30 \%)$ to one patient $(1 / 17,5.9 \%)$. Since instituting this regiment, it has been our observation that pin site infection has become a rare occurrence. We are not aware of any published literature that documents an infection rate as low as ours-the pin care protocol has made a significant impact on pin related complications.

The first paper discussing halo morbidity, published in 1986, advocated the use of povidone-iodine or hydrogen peroxide soaked gauze applied around pin sites to reduce infection rates [3]. A recent review advocates the use of chlorhexidene gluconate and alcohol over povidoneiodine solution as skin preparation [20]. This practice appears to have been efficacious in our series. There was no occurrence of eye injury or skin irritation with adherence to our protocol.

Loose pins were also a rare occurrence. While the initial correct placement is critical, keeping the tension in pins, checked on a regular basis, helped to reduce further loosening. All pins were tightened with a Bremer $0.90 \mathrm{Nm}$ wrench (DePuy Spine). One previous study has noted a higher torque for pin tightening $(0.68 \mathrm{Nm}$ vs. 0.90 $\mathrm{Nm}$ ), which resulted in lower pin site infections (36\% vs. $7 \%$ ) and loosening (20\% vs. 2\%) [21]. This has not been replicated elsewhere, but appears to have been efficacious in our series. There were no cases of skull fracture, penetration or cerebral abscess associated with higher torque values. Our population covered a wide age range with the oldest patient being 83. Concern has been expressed about the use of halo orthoses in older osteoporotic patients. While our sample size was small, there were no significant issues in our series. This finding has been recently noted elsewhere [22].

We realize and accept the limitations of a small retrospective case series and that ideally, our hypothesis would 
be tested by means of a large multicentre randomized controlled trial. We still feel that these results are significant and represent a comparative cohort study with sound basis and good results.

\section{Conclusions}

A significantly reduced incidence of morbidity, following halo application, can be achieved by utilizing a modified pin care regimen, focusing of pin asepsis and adequate torque.

\section{Conflict of Interest}

No potential conflict of interest relevant to this article was reported.

\section{References}

1. Lauweryns P. Role of conservative treatment of cervical spine injuries. Eur Spine J 2010;19 Suppl 1:S23-6.

2. Perry J, Nickel VL. Total cervicalspine fusion for neck paralysis. J Bone Joint Surg Am 1959;41:37-60.

3. Garfin SR, Botte MJ, Waters RL, Nickel VL. Complications in the use of the halo fixation device. J Bone Joint Surg Am 1986;68:320-5.

4. Tashjian RZ, Majercik S, Biffl WL, Palumbo MA, Cioffi WG. Halo-vest immobilization increases early morbidity and mortality in elderly odontoid fractures. J Trauma 2006;60:199-203.

5. Mercer M. Respiratory failure after tracheal extubation in a patient with halo frame cervical spine immobilization: rescue therapy using the Combitube airway. Br J Anaesth 2001;86:886-91.

6. Platzer P, Thalhammer G, Oberleitner G, Schuster R, Vecsei V, Gaebler C. Surgical treatment of dens fractures in elderly patients. J Bone Joint Surg Am 2007;89:1716-22.

7. Lind B, Bake B, Lundqvist C, Nordwall A. Influence of halo vest treatment on vital capacity. Spine (Phila Pa 1976) 1987;12:449-52.

8. Pande K, Basu S, Webb JK. Transient brain injury from penetration of a halo pin. Spinal Cord 1998;36:732-3.

9. Freidman RM, Rohrich RJ, Finn SS. Management of traumatic supraorbital neuroma. Ann Plast Surg 1992;28:573-4.
10. Rosenblum D, Ehrlich V. Brain abscess and psychosis as a complication of a halo orthosis. Arch Phys Med Rehabil 1995;76:865-7.

11. Saeed MU, Dacuycuy MA, Kennedy DJ. Halo pin insertion-associated brain abscess: case report and review of literature. Spine (Phila Pa 1976) 2007;32:E271-4.

12. Papagelopoulos PJ, Sapkas GS, Kateros KT, Papadakis SA, Vlamis JA, Falagas ME. Halo pin intracranial penetration and epidural abscess in a patient with a previous cranioplasty: case report and review of the literature. Spine (Phila Pa 1976) 2001;26:E463-7.

13. Hashimoto Y, Doita M, Hasuda K, Korosue K. Intracerebral pneumocephalus and hemiparesis as a complication of a halo vest in a patient with multiple myeloma. Case report. J Neurosurg 2004;100:367-71.

14. Kingma R, Peters JM, Coene LN. Intracranial penetration of a halo pin causing an epileptic seizure. J Bone Joint Surg Br 2006;88:1654-5.

15. Park P, Lodhia KR, Eden SV, Lewandrowski KU, McGillicuddy JE. Pin-site myiasis: a rare complication of halo orthosis. Spinal Cord 2005;43:684-6.

16. Dorfmuller G, Hollerhage HG. Severe intracranial injury from a fall in the halo external fixator. J Orthop Trauma 1992;6:366-9.

17. Davies R, Holt N, Nayagam S. The care of pin sites with external fixation. J Bone Joint Surg Br 2005;87:716-9.

18. Nemeth JA, Mattingly LG. Six-pin halo fixation and the resulting prevalence of pin-site complications. J Bone Joint Surg Am 2001;83:377-82.

19. Ruxton GD. The unequal variance t-test is an underused alternative to Student's t-test and the MannWhitney U test. Behav Ecol 2006;17:688-90.

20. Fletcher N, Sofianos D, Berkes MB, Obremskey WT. Prevention of perioperative infection. J Bone Joint Surg Am 2007;89:1605-18.

21. Botte MJ, Byrne TP, Garfin SR. Application of the halo device for immobilization of the cervical spine utilizing an increased torque pressure. J Bone Joint Surg Am 1987;69:750-2.

22. van Middendorp JJ, Slooff WB, Nellestein WR, Oner FC. Incidence of and risk factors for complications associated with halo-vest immobilization: a prospective, descriptive cohort study of 239 patients. J Bone Joint Surg Am 2009;91:71-9. 